\title{
Famiemprendimientos, desafíos de la mujer emprendedora
}

\section{Famiemprendimientos, challenges of the entrepreneurial woman}

Carolina de los Ángeles Uzcategui Sánchez

Ángel Zambrano Morales

Angeline Beltrán Vega

Universidad Metropolitana del Ecuador, Ecuador

Autor para correspondencia: carolina_uzcategui17@hotmail.com, azambrano@umet.edu.ec, angeline.beltran@udla.edu.ec

Fecha de recepción: 04 de Septiembre 2017 - Fecha de aceptación: 30 de Septiembre de 2017

Resumen: El presente artículo muestra una aproximación de tipo cualitativo sobre la motivación, características personales, lógica y dinámica de las mujeres emprendedoras de la ciudad de Machala, provincia de El Oro; además, se identifica la influencia del entorno familiar y social en el éxito del emprendimiento de la mujer; y, el involucramiento laboral de los miembros de la familia en las iniciativas de negocios, lo que se denomina famiemprendimiento. Se utilizó el estudio de caso como metodología para esta investigación, para lo cual, se llevó a cabo entrevistas a profundidad a 15 mujeres emprendedoras que fueron seleccionadas en función de las siguientes premisas i) el emprendimiento surgió bajo la iniciativa de una mujer, ii) el emprendimiento insertó laboralmente a por lo menos un integrante de la familia. Se muestran los resultados de acuerdo a tres dimensiones: i) características individuales de la mujer emprendedora, ii) factores que incidieron en el proceso de emprendimiento, iii) influencia del entorno familiar y social. En ese sentido, el famiemprendimiento en la ciudad de Machala es consecuencia de la necesidad de la mujer por superar dificultades familiares y sociales, además, las características personales y habilidades empresariales influyen significativamente en el éxito de las mujeres emprendedoras de Machala.

Palabras claves: famiemprendimientos; perdurabilidad; mipyme; mujer emprendedora; asumir riesgos

Abstract: This article presents a qualitative approach about motivational factors, personality traits and steps of the start-up process for women entrepreneurs in Machala city, El Oro province, in Ecuador. Family and social factors of influence in successful women's entrepreneurial ventures are also identified. Additionally, family labor involvement in business initiatives, known as famiemprendimiento, is also taken into account. The case study method was used for this research. As such, in-depth interviews were conducted with 15 female entrepreneurs who were selected based on the following criteria: i) the start-up venture emerged under the initiative of a female entrepreneur, ii) at least one member of the family was directly involved in the start-up venture as labor. Results are presented according to three dimensions: i) personality traits of entrepreneurial women, ii) factors that influenced the entrepreneurial process, iii) influence of family and social factors over the entrepreneurial process. In this sense, this research shows, famiemprendimiento in Machala city is a consequence of women's need to overcome family and social hardship. Furthermore, personality traits and entrepreneurial skills have a defining influence in the success of women as entrepreneurs in Machala.

Key words: family business; perdurability; start-up; women entrepreneurs; entrepreneurial skills 


\section{Introducción}

El término emprendedor, proviene del francés entrepreneur, que significaba estar listo a tomar decisiones o a iniciar algo (Segovia y Melgarejo, 2011; Rodríguez Ramírez (2009), precisa que el término ha evolucionado y se ha transformado con base a dos concepciones: la una caracterizaba por el individuo con capacidad guerrera que emprendía una lucha o hazaña, y reconocido por sus características personales; y la otra se refería al rey o jefe de Estado que planeaba sus estrategias y políticas para obtener éxito en sus metas, y era reconocido por sus funciones. Aunque en la actualidad, la mayoría de personas asocia el término a alguien que organiza y asume los riesgos en la ejecución de un negocio para la obtención de beneficios, definición propuesta por Cantillón (1755). Mientras que los libros de administración actuales, definen al término como la capacidad de iniciar y operar nuevas empresas (Brook, 1968; Bennis y Nanus, 1985; Mintzberg, Ahlstrand y Lampel, 1999; Vásquez, 2015), con características intrínsecas como el ser innovador, flexible, dinámico, capaz de asumir riesgos, creativo y orientado al crecimiento (Amit, 1997).

Desde el enfoque de género, en América Latina, el tema del emprendimiento va ganando espacio y relevancia (Álvarez et al., 2010; Amorós, Fernández y Tapia, 2011; Santander, Fernández y Yánez, 2016), destacándose estudios relacionados con los elementos que limitan la actividad emprendedora femenina (Moreira, Quintanilla y Verdesoto, 2016). En este punto, se debe diferenciar los dos tipos de emprendimientos: por necesidad (push) y los de oportunidad (pull) (Stoner y Fry, 1982), teniendo en cuenta lo anterior, se contrastan los resultados del GEM (2016), donde pese a que en América Latina, por cada 10 emprendedores masculinos, hay 8 femeninos; pero la mujer es más propensa a emprender por necesidad. Por lo cual, el emprendimiento femenino tiene menores probabilidades de tener éxito y perdurabilidad en el tiempo (Kelly et al., 2015).

Además se puede indicar que el tema ha tomado matices específicos derivados de la evolución del rol que desempeña la mujer a nivel social, familiar y laboral (Briseño, Briseño y Lira, 2010). Es así que la mujer se ve obligada a insertarse en el mercado laboral de forma independiente (emprender) como consecuencia de sus obligaciones de género en el hogar o por la falta de oportunidades derivadas de la misma causa (Castiblanco, 2013; Rodríguez y Rodríguez, 2011). El emprendimiento de la mujer se potencializa o condiciona por la influencia de determinados factores, como: a) las influencias anteriores, entendidos como las influecias de la familia, factores genéticos, stock de habilidades y conocimiento; b) interacción con incubadora, donde por la naturaleza de la empresa, el emprendedor ha desarrollado sus actividades empresariales; y c) factores del contexto, entendidos como condicionantes económicas, acceso a fuentes de financiamiento y disponibilidad de servicios soporte (Jia, Pavin y Rahman, 2012).

Tomando en cuenta lo anterior, el presente estudio trata de brindar una aproximación cualitativa, sobre el emprendimiento de la mujer y adicionalmente se incluye el involucramiento de los miembros de la familiar laboralmente en iniciativas de negocios exitosos, configuración que se la ha denominado famiemprendimiento. La concepción del análisis se sustenta en tres dimensiones, la dimensión individual, la contextual y la del proceso de emprender (Gartner, 1985; Gartner, 1988; Bolaños, 2006). 


\section{Metodología}

El presente estudio es una investigación cualitativa de corte estudio de caso y con enfoque de género, donde se realiza una aproximación cualitativa sobre la lógica, motivación y dinámica (Íñiguez Rueda, 1999), de mujeres emprendedoras de Machala que involucraron a diversos integrantes de su familia en las actividades operativa o empresariales del negocio. La utilización de la metodología cualitativa brinda una visión holística, comprensiva y contextualizada del fenómeno estudiado (Olabuénaga, 2012). La recolección de datos se efectuó a través del método de la entrevista, que facilita al investigador explorar de forma profunda aspectos subyacentes de la temática investigada (Chárriez Cordero, 2012), la entrevista fue de tipo narrativa, caracterizada por poner su foco de atención en las historias que las entrevistadas cuentan de su propia vida (Kvale, 2011; Chárriez Cordero, 2012).

La identificación de potenciales entrevistadas fue la etapa final de un proceso que tuvo como fases previas la búsqueda y selección de las candidatas, que se llevó a cabo entre el 15 de mayo al 30 de junio de 2017; para la búsqueda de participantes se la realizó de forma intencional y también se utilizó la técnica bola de nieve. El criterio de partida fue que el emprendimiento haya sido propuesto por una mujer, el número de participantes no fue preestablecido, la cantidad encontrada de potenciales participantes fue de 50, adicional con la información levantada se construyó una base de datos de contacto, que incluyó nombre de mujer emprendedora, nombre del negocio, dirección, teléfono, e-mail. Mientras que para la selección, se filtró las candidatas en función del cumplimiento de dos premisas: a) el emprendimiento surgió bajo la iniciativa de una mujer; y b) el emprendimiento insertó laboralmente a por lo menos un integrante de la familia.

El total de entrevistadas seleccionadas fue de 15 candidatas, de las cuales colaboran casi todas a excepción de una (debido a dificultad de convenir una cita de contacto). La entrevista a profundidad como tal, se la realizó a través del contacto personal para facilitar interlocución entrevistador-entrevistada (Perera, 1995). Tras la presentación inicial de la investigadora, se presentó a las entrevistadas el objetivo del estudio y se les explicó sobre el modo de recolección de datos; luego se aplicó una guía de entrevista con preguntas abiertas, que incluyeron las siguientes dimensiones, según Gartner (1988): individual, contexto y proceso. También, se aseguró confidencialidad de datos personales, se recogió una autorización firmada de entrevistadas y el registro se lo realizó a través de una aplicación de celular para grabación de voz. Las entrevistas tuvieron una duración entre $25^{\prime}$ a 45' y se las trascribió verbatim.

\section{Dimensión individuo}

\section{Resultados}

Dentro de la dimensión individuo se encontró que los factores motivacionales y experienciales declarados por las entrevistadas sobre el surgimiento de su idea de negocio son los siguientes: 1) necesidad económica debido a la falta de un trabajo apropiado a sus capacidades y requerimientos personales y familiares, 2) capacidad de detectar oportunidades para crear nuevos productos y/o servicios, 3) habilidades y destrezas adquiridas a lo largo de su formación formal e informal, 4) experiencia laboral previa, 5) independencia económica y sentido de logro, 4) tradición familiar de crear su propia empresa 
En efecto, la mayor parte de las participantes coincidieron en sus respuestas al referirse a las cualidades para iniciar un emprendimiento familiar que las llevó a creer que tendrían éxito, entre las que describieron, se resalta las características personales como la responsabilidad, visión, capacidad para resistir y sobrellevar situaciones difíciles entre otras esenciales para continuar con el negocio. Otros aspectos importantes están relacionados con el talento, habilidades y destrezas además de los valores familiares arraigados en cada emprendedora.

\section{Dimensión proceso}

Con respecto a la dimensión proceso de emprendimiento las entrevistadas enfatizaron que los desafíos personales y laborales que enfrentaron con mayor recurrencia fueron encontrar el equilibrio entre familia y negocios, serias dificultades para acceder a créditos en las diferentes instituciones financieras y el incremento de la competencia. Finalmente indicaron que las constantes críticas de su círculo de amistades y en ciertas ocasiones de su familia en la etapa de inicio y puesta en marcha su empresa, provocaron muchas veces desánimo; sin embargo, esto último, lo interpretaron como parte natural de asumir cambios y riesgos en el contexto de nuestra sociedad.

Las entrevistadas también aseguraron que los factores de éxito para un emprendimiento familiar, giran en torno a responder de forma efectiva las necesidades de los clientes, sumado al apoyo familiar dentro de los procesos organizacionales, además de proyectar seguridad y confianza en sí mismas para liderar la empresa con perseverancia y objetividad, y lidiar con los fuertes condicionamientos derivados de su preparación y experiencia laboral previa.

\section{Dimensión entorno}

El entorno donde se desarrollan las emprendedoras es la tercera dimensión a enfatizar, debido a que muchas participantes relataron la forma como administran sus empresas y la influencia del apoyo familiar que recibieron, desde esta perspectiva, manifestaron que la convergencia de las relaciones laborales y familiares se compagina mediante la separación y cumplimiento de los roles laborales asignados, sumado a los valores familiares compartidos, entre ellos el compromiso al demostrar mayor flexibilidad y disponibilidad para el trabajo por cada familiar vinculado al negocio lo que genera la sinergia necesaria para la toma de decisiones claves.

En relación a las ventajas de trabajar en familia las emprendedoras señalaron de forma categórica la relación solidaria o de apoyo mutuo, el grado de compromiso asumido, esfuerzo y dedicación y el ambiente de confianza y de comunicación que se pueda dar; sin embargo, aparecen también las desventajas asociadas a los desacuerdos que pueden existir en la forma de administración causada por la inadecuada asignación de funciones, dificultad para elevar los niveles de exigencia con los integrantes de la familia lo que provoca incomodidad laboral y la ruptura de la relación familiar.

\section{Conclusiones}

Los resultados de la investigación cualitativa enmarcados en las dimensiones del proceso de emprendimiento indican que las mujeres de Machala crean empresas por necesidad 
económica ante la falta de opciones laborales apropiadas que se ajusten a los requerimientos personales y familiares, además otro factor clave se refleja en el estímulo y participación de miembros de su familia como elemento clave de su labor con la finalidad de transformar sus vidas y sentir la satisfacción de obtener mayor independencia y control en el ámbito social y económico.

En efecto, el éxito alcanzado por las emprendedoras se sustenta no solo en sus talentos, habilidades y destrezas adquiridas sino más bien en sus características personales como la proactividad, visión y un alto sentido de responsabilidad sumado a grandes dosis de resiliencia indispensables para desarrollar su actividad empresarial familiar.

Las mujeres fundan sus empresas familiares para la subsistencia de su familia, de ahí la necesidad de enfrentar desafíos como extensas jornadas de trabajo como requisito para garantizar la permanencia de la empresa, un gran número de mujeres coinciden en eso, al mencionar de sus esfuerzos para conciliar y equilibrar la vida familiar con las demandas de la empresa propia; sin embargo y pese a las dificultades encontradas en el trayecto para emprender, la más relevante constituye el entorno hostil en el que se vieron expuestas tanto por sus iniciativas innovadoras como por su nivel de empoderamiento de sus ideas de negocio, dicho ambiente originado desde su círculo de amistades y familiares más próximos sumado al hecho de la percepción de la sociedad que muchas veces ve con escepticismo que una mujer pueda tener éxito empresarial bajo las mismas circunstancias que los hombres.

En consecuencia, una emprendedora potencialmente exitosa es aquella que cuenta con el capital generado especialmente por las relaciones solidarias de consenso y apoyo familiar; y que proyecte una personalidad de confianza en sí misma, seguridad, perseverancia y liderazgo para sacar adelante su empresa.

Finalmente, aquellas mujeres, que día tras día, han llegado a cumplir sus sueños y objetivos, a través de prácticas estas prácticas empresariales consistentes junto a su familia, se convierten en un referente para el resto de mujeres sobre la necesidad de emprender, la asunción de riesgos y la búsqueda de un mejor nivel de vida, propio y de los demás.

\section{Agradecimiento}

Se ofrece un sincero agradecimiento a la Universidad Metropolitana, por brindar el apoyo a la investigación a través del proyecto de investigación "Propuestas de mejora de la competitividad empresarial de la mipyme de la provincia de El Oro", este artículo emerge como un resultado parcial dentro del conjunto de actividades del proyecto. También se agradece de forma distinguida a la Ing. Angeline Beltrán, MSc. por las sugerencias, correcciones y colaboración en la finalización del artículo.

\section{Bibliografía}

Álvarez, A., Valencia de Lara, P., Barraza, S., \& Legato, AM. (2010). Factors determining the entrepreneurial consolidation in Latin America. African Journal of Business Management, 4 (9), 1717-1722 
Amit, R. (1997). Charla de intraempresario. Conferencia Internacional. Sydney, Australia.

Amorós, J. E., Fernández, C., \& Tapia, J. (2011). Quantifying the relationship between entrepreneurship and competitiveness development stages in Latin America. International Entrepreneurship and Management Journal. DoI: 10.1007/s11365-010-0165-9

Bennis, W. \& Nanus, B. (1985). Leaders: the strategies for taking charge. New York: Harper and Row Publisher.

Bolaños, R. (2006). Detonantes de emprendimiento. VIII Reunión de Economía Mundial. Tecnológico de Monterrey, Campus Estado de México. www.pyme.com.mx

Briseño, O., Briseño, A., \& Lira, A. (2016). El emprendimiento femenino: un estudio multi-caso de factores críticos en el noreste de México (Female entrepreneurship: a multi-case study of critical factors in Northeastern Mexico). Innovaciones de Negocios, 13(25), 23-46.

Brook, P. (1968). The empty space. Paperback Publisher. USA.

Cantillón, R. [1755]. Essai sur la nature du commerce en general (H. Higgs, Ed.and transT). London: Macmillan, 1931.

Castiblanco, S. (2013). "La construcción de la categoría de emprendimiento femenino". Revista Facultad de Ciencias Económicas: Investigación y Reexión. Vol. 21, No. 2. Bogotá, Colombia. Pp. 53-66. Disponible en http://www.redalyc.org/pdf/909/90930501005.pdf

Chárriez Cordero, M. (2012). Historias de vida: Una metodología de investigación cualitativa. Revista Griot (Etapa IV-Colección completa), 5(1), 50-67.

Gartner, W. B. (1985). A conceptual framework for describing the phenomenon of new venture creation. Academy of management review, 10(4), 696-706. Disponible en http://www.elitaschillaci.it/Didattica/Gartner.pdf

Gartner, W.B. (1988). Who is an entrepreneur? Is the wrong question. Entrepreneurship Theory and Practice, 13, Summer, 47-68. Disponible en https://zabdesk.szabist.edu.pk/CoursePortFolioFiles/Mubin_1140_2758_1/who\%20is\%20 an\%20ent\%20-\%20lums.pdf

GEM. (2016). Report 2016. World Health Organization, Geneva. Retrieved in http://www.gemconsortium.org/report

Íñiguez Rueda, L. (1999). Investigación y evaluación cualitativa: bases teóricas y conceptuales. Recuperado en http://www.elsevier.es/es-revista-atencion-primaria-27-articuloinvestigacion-evaluacion-cualitativa-bases-teoricas-14823 
Jia, J., Parvin, L. \& Rahman, M. (2012) Determinates of women micro-entrepreneurship: An empirical investigation in rural Bangladesh. En: International journal of Economics and Finance, 4 (5). Disponible en http://ccsenet.org/journal/index.php/ijef/article/view/16528/0

Kelley, D., Brush, C., Greene, P., Herrington, M., Ali, A. y Kew, P. (2015). Special report: Women's entrepreneurship. World Health Organization, Geneva. Recuperado en http://www.womenable.com/content/userfiles/gem-2014-womens-report.pdf

Kvale, S. (2011). Las entrevistas en investigación cualitativa. Ediciones Morata.

Mintzberg, H., Ahlstrand, B. \& Lampel, S. (1999). Safari a la estrategia. Argentina: Ediciones Granica.

Moreira, C., Quintanilla, J., y Verdesoto, O. (2016). Influencia del género en el desarrollo del emprendimiento. Revista Publicando, 3(8), 295-316. Disponible en: http://www.rmlconsultores.com/revista/index.php/crv/article/view/263

Olabuénaga, J. I. R. (2012). Metodología de la investigación cualitativa (Vol. 15). Universidad de Deusto.

Perera, S. (1995). Las condiciones de interacción comunicativa en las técnicas lingüísticas de obtención de información y sus efectos en la elaboración discursiva sobre el cuerpo y la educación. Universitat de Girona.

Rodríguez Ramírez, A. (2009). Nuevas perspectivas para entender el emprendimiento empresarial. Pensamiento \& Gestión, (26), 94-119. Disponible en http://rcientificas.uninorte.edu.co/index.php/pensamiento/article/viewFile/864/508

Rodríguez, M., y Rodríguez, M. (2011). La mujer en la empresa familiar. Disponible en http://helvia.uco.es/xmlui/handle/10396/8757

Santander, P., Fernández, C., y Yáñez, D. (2016). Motivaciones y condicionantes contextuales en el emprendimiento liderado por mujeres chilenas. Revista de Ciencias Sociales (Ve), 22(2), 63-77. Disponible en http://www.redalyc.org/pdf/280/28049145006.pdf

Segovia, A., y Melgarejo, Z. (2011). La famiempresa, un sistema vivo y abierto de desarrollo socioeconómico. Tendencias: Revista de la Facultad de Ciencias Económicas y Administrativas, 12(2).

Stoner, C. y Fry, F. (1982). The entrepreneurial decisión: dissatisfaction or opportunity? Journal of Small Business Management. 20 (2), 39-44.

Vásquez, J. (2015). El Emprendimiento Empresarial: La Importancia de ser Emprendedor. IT Campus Academy. 